small a number of cases, all of these arguments are intense; face much swollen, so that the eyes were parrefuted by the cases which came under my observa- tially closed; œdema of hands; urine contained a tion. trace of albumen and a few casts. On third and

In only one case did the diphtheritic inflammation appear early in the course of the scarlet fever; in the others the membrane was observed on the third or fourth day, or during the stage of desquamation.

One case died from invasion of the larynx by the diphtheritic membrane. In a second case there was probably membranous laryngitis, as evidenced by hoarseness and embarrassed respiration. The child died apparently from asthenia. I doubt if tracheotomy would have been of any avail, even if the parents had assented to the performance of the operation.

Two cases died of paralysis of the heart; one when convalescence was perfectly established, and one when the child was apparently improving rapidly.

The mortality was large, as four out of eight $(50 \%)$ died.

Cases of especial interest are the following :

Millie Hackett, three years. When first seen there were remains of a rash of scarlet fever; on both tonsils and uvula a dirty gray membrane, which spread in a day or so to the pillars of the fauces. Parts bled if touched. Another physician was called, as my prognosis was grave. On the eleventh day I saw the child playing in the street. No ulceration in the pharynx. On twelfth day, sudden death from paralysis of the heart, according to the account given by the family.

Johanna Molineux, two and a half years. Brother of the patient had had a mild attack of scarlet fever eleven days previously. Patient had a mild attack without marked pharyngitis. Fifth day, tonsils much swollen. In the course of a few days both tonsils and uvula were covered with membrane; lips and edges of nostrils ulcerated and bleeding. Thirteenth day, voice hoarse and almost inaudible; respiration somewhat embarrassed. Fifteenth day, death, without any preceding dyspnœa. Trace of albumen and a few casts in the urine.

Daniel Shields, five years. When first seen desquamation was going on; emaciation extreme; voice whining; ulceration of the edges of lips and nostrils; membrane on both tonsils and uvula; abscess below right ear that contained thin, bloody, foul-smelling pus. Treatment: locally, iodoform; internally, corrosive sublimate (gr. $\frac{1}{60}$ every hour), whiskey and eggs. Perfect recovery.

Three cases occurred in one family by the name of O'Brien. Hygienic surroundings good.

September 14th. Louisa, eleven years. Malaise; slight pharyngitis; rash, which lasted twenty-four hours. Second day, perfectly well. No sequelæ. No desquamation.

September 14th. Mary, nine years. Eruption very full and bright. 'Third day, membrane on uvula and pillars of the fauces; mucous membrane of lips ulcerated and bleeding. In the course of a few days, foul ulcer on right elbow, ulcer of genitals and perinæum. Sixth day, right knee red, swollen and tender. Next day same symptoms in left knee and left wrist. The disappearance of the latter symptoms in two days, under the use of salicylic of sodium made the diagnosis of rheumatism probable. Sudden death on tenth day.

James, three years, and Anne, four years. Both taken sick September $17 \mathrm{th}$. In both cases eruption

fourth days respectively a membrane appeared on the tonsils and uvula. Both recovered completely.

The œdema in these cases was apparently due to the intensity of the eruption, as in neither case did the examination of the urine warrant the diagnosis of Bright's disease. Two other children that slept in the same room were not sick. It is an interesting fact that during the height of the disease the mother was confined, and convalesced without having any puerperal fever. She had no doctor or nurse.

\title{
SUMMARY.
}

Of these fifty cases, four died (one in twelve), all of diphtheria. These four cases are the only ones that died in my district, of scarlet fever; from April to January, the total number of cases being eighty-eight. This gives a mortality of one in twenty-two.

It has been interesting to me, and very satisfactory, to find that I have not been called to a single case of scarlet fever in a family which $I$ had attended previously for any other disease. Of course it was impossible for me to think of changing my clothes, or even disinfecting the hands, . before attending other cases.

The new cases visited each month average in my district two hundred.

The eight and only cases of diphtheria, all occurred in three weeks, and represented one-third of the total number visited in the three weeks.

Treatment. In mild cases expectant. In cases with high fever, tepid sponge baths and autipyrine. In cases with diphtheria, brandy and tinctura ferri chloridi.

\section{TYPHOID AND MALARIAL FEVER IN BOSTON.}

BY JOHN A. JEFFRIES, M.D., BOSTON.

IN view of the recent article on typho-malaria in Boston, by Dr. Whittier, the following cases seem of interest. They all occurred in an area bounded by the water, Kneeland, Kingston, and Essex Streets. The first two cases are examples of quotidian and tertian malaria, and are given as the histories seem to suggest infection contracted in Boston. The rest of the cases all occurred in one family, two of whom had typhoid fever at the same time. Both the relapse of malaria and the typhoid seemed to be due to crowding in two sunless, dirty, badly drained and ventilated rooms.

Case I. April 28, 1887. J. H., male, twenty-six years old, married; has always lived in Melrose until last winter, when he moved into his present lodgings. Always healthy; knew nothing of malaria. When first seen he complained of being seized with fever, vomiting, and pain in the abdomen, about six in the morning. Now in a profuse sweat; temperature in the axilla, $103.6^{\circ}$, pulse 120 , respiration 20 . All the viscera examined, and nothing abnormal found. Urine clear, light, profuse; no albumen. Temperature at 5 P. M., $99^{\circ}$, pulse 80 .

April 29th. Had a fever early this morning; temperature at $11 \mathrm{~A} . \mathrm{M} ., 101^{\circ}$; at 5 P. M., $99^{\circ}$.

April 30th. Had a fever same as the last two days; temperature at 11 A. M., $102^{\circ}$. Still no physical signs. Quinine ordered at night. 
No more chills occurred, and patient went to work in two days.

Case II. September 12, 1887. D. C., male, twentyfour years old, married. Born and bred in Paris, went to New Orleans for a week, and then came to Boston six months ago; never had a fever or periodic disease. At the time of my visit patient seemed unstrung, and gave a history of being waked up early in the morning by a hard chill, followed by fever and sweating. No physical signs.

September 13th. Seems perfectly well.

September 14th. Had a chill again this morning, with fever and sweating; still sweating at time of visit. Ordered a full dose of quinine four hours before the time of the next attack. From this time on no chills occurred, but patient was poorly for ten days, with a febrile rise of temperature on the even dates.

Case III. August 8, 1887. G. D., male, seven years old, pale and thin ; old history of tertian malaria in Springfield, two years ago. Now suffering from mild diarrhœa. Prescribed camphorated tincture of opium and bismuth.

August 9 th. No diarrhœa, but child the same.

August 10th. No diarrhœa, but child has tuken to his bed, and mother gives history of a chill about 9 A. M. Physical examination showed head and chest normal; the liver to be felt at the edge of the ribs on the right side, and a half-inch below the ribs on the left side; spleen out to ends of ribs, tense, tender; bowels not tender, gurgling.

From this time on the child ran a regular course of alternate poor and bad days, vomiting, numerous ochre stools, bronchitis, and prostration being the constant symptoms, and chills on alternate days. On the seventeenth prostration was great, and much stimulants had to be given. Convalescence was slow, rose-spots were not seen. The treatment was confined to quinine, milk, raw eggs, and whiskey. Below is given a temperature chart, from which it will be seen that during the collapse the malaria changed from the even to the odd dates.

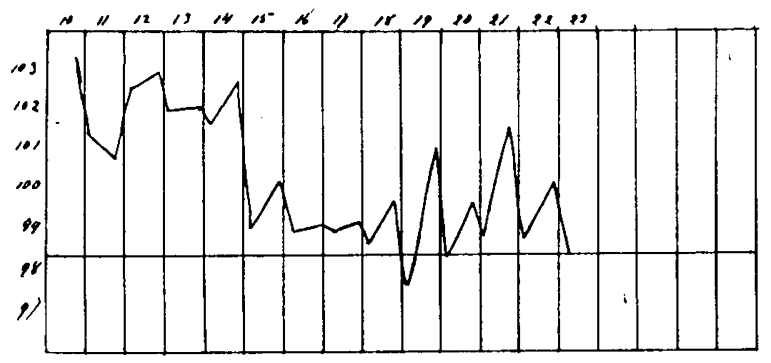

Case IV. August 10th. F. D., male, sixteen months old, brother of Case III. Caught malaria, when four months old, in Springfield. Had a distinct chill and fever, no sweat noted, on the $9 \mathrm{th}, 11 \mathrm{th}$, and $13 \mathrm{th}$. Treatment, quinine.

Shortly after, while the boy was still sick, the mother came down with tertian chills, and had to take much quinine. Spleen enlarged.

Lastly, on the 10th of September, the father came down with a mild attack, like Case IV, of which I was unable to secure any satisfactory records.

The first two cases are reported as examples of pure malaria, occurring for the first time in men who had for at least six months been resident in Boston. The third case is a very fair example of typho-malaria, or, as I prefer to call it, and reported to the Board of Health, typhoid and malaria. It will be noted that the malaria was a relapse of an old trouble, apparently induced by the same lack of sanitary conditions that caused the two cases of typhoid.

All my other cases of typhoid fever ran a regular course, showing no sudden changes from day to day.

\section{PROGRESS IN THE CARE OF THE INSANE.}

BY Walter chanNiNg, M.D.

\section{PROVISION FOR THE INSANE.}

THe year 1887 has shown further and important progress in correct methods of providing hospital or asylum accommodations for the insane.

'The theory that alms-houses can be so managed as to properly care for and treat insane persons, has been strenuously and persistently combatted, and in time such an inadequate, not to say reprehensible means of provision must be wholly done away with.

Strong arguments in favor of placing the insane entirely in the care of the State have recently been advanced by the American Journal of Insanity. The Journal quotes the following, in its January number, from a letter of the Hon. Wm. P. Letchworth, President of the New York Board of State Charities : "I believe there is no question but that State care is better than county care; and when the cost is not estimated, but arrived at by a separate system of accounting, is cheaper. Several counties have tested this, and found to their surprise that their insane cost them much more kept at home than if sent to Wiiliard (a large asylum for the chronic insane). ... The reason why State care is better lies in the fact that it is administered by uncompensated, nonpartisan boards, whose members are employed for long terms. Boards of supervisors are constantly changing their membership, and the great advantage arising from cumulative experience is lost. Besides, under the county system, the care of the insane becomes, to a greater or less degree, a medium for the distribution of political patronage."

Dr. P. M. Wise, superintendent of the Williard Asylum, reiterates what he has often said before, that each year's experience proves that the county care of the insane is liable to relapse into a condition far below a proper standard, and the remedy is not always within reach. ${ }^{1}$ Dr. Wise quotes Dr. Henry M. Hurd, of Michigan, who says: "The county system, as a whole, lacks definiteness of plan, and is hap-hazard and capricious, depending too much upon local public sentiment and individual ideas of reform and economy." 2

Taking the experience of nearly all observers of the last thirty years, it may be stated as a settled fact that State care for the insane is safe, county care hazardous, and generally unsafe, and any further steps taken in providing for the insane should be made clearly and systematically with this idea in view. Any exception, any departure from this plan, however attractive and easy at the moment, will be pretty sure to be attended by unfortunate consequences in the end.

1 Annual Report, 1887.

2 Colony System of Michigan. 